\title{
Inclusive Accommodations for Persons with Visual Impairments in Computer- Based Tests
}

\author{
Pawan Kumar Patel ${ }^{\mathrm{a}}$, Amey Karkare ${ }^{\mathrm{a}}$ and Gaurav Raheja ${ }^{\mathrm{b}}$ \\ ${ }^{\mathrm{a}}$ Indian Institute of Technology Kanpur \\ b Indian Institute of Technology Roorkee
}

\begin{abstract}
The accommodations in exams (for example, scribe, compensatory time, and magnification) are widely used for many years to accommodate persons with visual impairments (PVIs). Nowadays, most of the exams are conducted using computers and web-based technologies, referred to as Computer-based tests (CBTs). These CBTs play an important role in the professional assessment of an individual, starting from university admissions, courses evaluation, and grading, to recruitment in various sectors like banking, software, railways, etc. Barriers in accessing certain components of the CBTs limit the utilization of Computer-based technologies for PVIs. In this research, the availability and effectiveness of common accommodations in CBTs were evaluated and reviewed. To the best of our knowledge, this is one of the early attempts from India to systematically study the effectiveness of these accommodations. Driven by a universal design approach, it can play a key role towards the development of an inclusive examination system.
\end{abstract}

Keywords. Inclusive Accommodations, Accessibility, Computer-Based Test (CBT), Persons with Visual Impairments (PVIs)

\section{Introduction}

As per census 2011 India, 45\% of persons with disabilities in India are uneducated. Computer-based tests (CBTs) are critical gateways to access higher education, employment for individuals in India, a country having the challenge of very large population. Starting from getting admission into a prestigious institution up to procuring a well-paid job, an individual must pass through several phases of highly competitive examinations. The mode of conducting exams is gradually shifting from traditional penpaper based tests to CBTs due to the increasing number of applicants, and to achieve objectivity in evaluation procedures and to reduce the vulnerabilities involved. However, inclusion in the examination and the assessment procedures still remains a critical area to enable access for education or jobs for persons with disabilities in India. Universal design approach could build a holistic understanding of inclusion through CBTs, thereby promoting equity and access in education and jobs as the larger goal. Many children of India remain illiterate owing to extreme disconnects between educational environments and their method of evaluation. This study is limited to persons with visual impairments (PVIs), even though the qualitative analysis for other disabilities was also done. 
Utilization of technologies in CBTs is quite confined for PVIs due to their limitation to perform certain physical activities, for instance, reading regular fonts, low color contrast text, and many more, as presented in an earlier study [1]. This makes certain components of the CBTs partially or completely inaccessible. In order to extend equal opportunities, same as temporary able-bodied individuals, to PVIs, it is essential to provide inclusive accommodations to PVIs.

Some CBTs do have provisions for providing accommodations to PVIs to mitigate the accessibility barriers during the exams. In India, the most commonly used accommodations in CBTs are:

- Magnification of the text.

- Provision of a scribe to assist in reading and writing.

- Compensatory or Additional time to complete the examination.

Above mentioned accommodations are in use for many years in India and are part of Government of India guidelines for conducting written examination for persons with disabilities ${ }^{1}$. However, to the best of our knowledge, this is one of the early attempts to systematically study the effectiveness of these accommodations. It can play a key role towards the understanding and the development of an inclusive examination system.

This paper evaluates the diverse possibilities of inclusive accommodations used in CBTs, highlighting the specific challenges faced by PVIs. Based on the experimental survey of various accommodations, it discusses the various universal design implications and recommendations for inclusive accommodations in CBTs.

The paper is organized as follows: Section 2 below presents related work to the study. Section 3 below provides a detailed description of the research methodologies used in the study. Section 4 describes the data, its findings and extends into a discussion in Section 5 to propose few related recommendations. The paper concludes in Section 6 with possible directions for future work.

\section{Related Work}

Assistive technology plays a key role in enhancing the accessibility of CBTs, as presented in a research study [2]. Although technology advances have enabled the access of unlimited educational resources to all, including PVIs, there are plenty of barriers to access CBTs. The authors describe that in order to overcome the accessibility barriers in CBTs, technical accessibility standards should be created. Hence, to evolve these standards and guidelines related to the accessibility of CBTs, empirical studies about the accessibility of CBTs need to be conducted. The author has given an overview of accessibility barriers in CBTs, while our empirical study presents the accessibility barriers in CBTs along with the effectiveness of various accommodations provided to PVIs.

A typical recruitment process involves many steps from the job search to interview. A research study [3] presented the accessibility barriers in eleven complementary aspects of a classic recruitment process, starting from the job search, application, exams, interview until on-boarding. The accessibility challenges in each step of a job recruitment process have been highlighted in this study of eight PVIs from a technological background. The CBTs are a key component for final recruitment in a

${ }^{1} \mathrm{http} / /$ www.disabilityaffairs.gov.in/content/page/guidelines.php 
typical setting. Hence inclusive accommodations become critical for the success of PVIs. The authors also highlighted the unawareness of examination authorities about the availability of inclusive accommodations for persons with disabilities during the exam. This paper gives an overview of the accessibility concerns of all the components involved in the recruitment process. On the other hand, our study is a detailed, in-depth analysis of a single component, i.e., (computer-based) exams.

In educational institutions, persons with disabilities need to perform more administrative tasks as compared to temporary able-bodied, such as to fill inclusive accommodations form, to get inclusive accommodations letter, etc. Accessibility barriers associated with such administrative processes were presented in the study [4]. The authors had analyzed the level of difficulty of every single administrative process and reported the impacts on the individual. The authors have talked about the barriers in requesting various adjustments in studies, examinations, and day-to-day activities (such as travel and parking), but, unlike our work, they do not go into the details of specific barriers in the examinations themselves.

Brajnik and Graca [5] presented accessibility policies as a fundamental instrument for implementing accessibility solutions. They surveyed the accessibility policies of twenty universities in Europe and highlighted the need for the development of accessibility policies in higher education institutions to cater to the needs (including exam-related needs) of persons (students, staffs, and faculties) with disabilities. The basis for measuring the comprehensiveness and concreteness of policy includes three parameters - role, content, and quality of the policy. In the policy-making scenario, authors reported that web-development, and hence web developers to be one of the important components.

The study presented in our paper complements the studies mentioned in [3], [4], and [5]. CBTs are important components of examinations in educational institutions as well as of the recruitment process. Therefore, technical accessibility standards, accessibility policies pertaining to $\mathrm{CBTs}$, accessible administrative processes, and eventually accessible recruitment processes are integral to the professional success of persons with disabilities.

\section{Methodology}

Google form comprising of single choice, multiple-choice, Likert scale type, and short answer type questions was created to assess the accessibility barriers in the CBTs. The participants were allowed to fill the past experiences of up to three online examinations in a single form ${ }^{2}$ anonymously. The complete questionnaire and the raw data collected are available on the project page ${ }^{3}$.

${ }^{2}$ We allowed data for up to three exams to keep a balance between diversity of exams and candidates. Any participant could have filled multiple forms to give data about more exams, but we cannot know about it due to the anonymity of participants.

${ }^{3}$ https://www.cse.iitk.ac.in/users/karkare/accSurvey. 


\subsection{Survey Design}

The questionnaire was divided into three major sections:

- General information about the participants: Information about age, disability type, and its extent was included in this section, along with the objective of the research study and electronic consent for voluntary participation. To facilitate the complete anonymity of individual participants, we did not collect any personal information about the participants (such as. email, phone number, address, profession). The anonymity allows participants to give honest feedback about the availability and expected inclusive accommodations in the CBTs. Information related to gender was not collected since neither the impairments nor the inclusive accommodations in the examinations are based on gender.

- CBT experience without accommodation: In order to discover the holistic picture of accessibility barriers in the CBT interfaces, participants were asked to share their experiences about accessibility of CBT interfaces without the use of accommodations. The major questions include the name of the examination, the extent of the readability of the questions, reading upper case words, reading subscripts/superscripts, accessibility of different components, and reasons behind limitations to read the questions.

- CBT experience with available accommodations: This part was designed to assess the effectiveness of the currently available accommodations in CBTs. Participants were asked about what accommodations were available during the CBTs, their preference for specific inclusive accommodations, the effectiveness of commonly available accommodations, their preferred way of reading text, and the overall rating of CBT interface on a Likert scale of 0 (Very poor) to 5 (Excellent).

\subsection{Data Collected}

This study was confined to competitive CBTs in India. Although geographical information about the participants was not gathered, there was still sufficient diversity in the pool of participants. The pool of participants from national forums includes educational institutes like IIT Kanpur ${ }^{4}$, IIT Delhi ${ }^{5}$, and registered participants at events including Empower $2018^{6}$ and I-STEM Hackathon 2018 ${ }^{7}$. These institutions and events have the participation of persons with disabilities from all over India. Invitations were sent to registered email ids of persons with disabilities of the above-mentioned events and institutions. Responses from 24 participants were received by the deadline prior to (January $15^{\text {th }}, 2019$ ), out of which one response was ignored due to an invalid entry in the field 'name of examination appeared in'. In the remaining 23 responses, six participants have shared their experiences in two different CBTs, one participant filled the survey for three different CBTs, and the remaining 16 participants had filled the questionnaire for only one CBT.

\footnotetext{
${ }^{4} \mathrm{http}: / /$ www.iitk.ac.in

${ }^{5} \mathrm{http}: / / \mathrm{www}$. iitd.ac.in

${ }^{6} \mathrm{http}: / /$ assistech.iitd.ac.in/empower2018

${ }^{7} \mathrm{http}$ ://inclusivestem.org/hackathon-2018.html
} 


\subsection{Data Preprocessing}

The very initial step was to clean the data. We followed a semi-automated approach using scripts developed in Python 2.7 along with little manual intervention. Below are the steps followed for data preprocessing:

- Each exam was considered as a separate entry (row) for study purposes. For participants who shared experiences for more than one CBT, general information was duplicated for each $\mathrm{CBT}$, thus resulting in a single row for each participant for each CBT. This simplified the analysis of the data while maintaining completeness and correctness.

- Rows having an invalid response to 'Name of Online Exam you appeared in' were ignored straight away since these rows defeat the core purpose of the study.

- Entries that did not satisfy the conditional dependency on a previously asked question were discarded as they could distort the actual result. Below is an illustration of discarding such entries:

○ Did you get the magnification enabled in the exam?

○ If you have answered "yes" to the previous question. Please let us know to what extent are you able to read the questions/instructions during the exam after enabling the magnification?

Consider the above scenario where the second question is dependent on the response of the first question. For a participant responding a 'no' or 'not applicable' to the first question, any response in the second question was considered as 'not applicable'.

\subsection{Data Analysis}

Python 2.7 modules including pandas, NumPy, statsmodels, matplotlib, and seaborn were used to analyze the preprocessed data. Since the participants who responded were all PVIs except one with cerebral palsy, we performed the analysis for various dimensions of the visual impairment disability. We made several observations related to the various challenges faced by specific ranges of the extent of disability. Further, a comparison between available accommodations versus expected accommodations in CBTs was also performed.

\section{Findings}

The section presents observations and findings of this empirical study based on the responses received until January $15^{\text {th }}, 2019$. Analysis for dimension 'extent of disability' was performed using two different scales: one below $60 \%$ and another at four regular intervals from $60 \%$ to $100 \%$. However, since we did not receive any responses in the range of $80 \%-90 \%$ disability, the category $80 \%-90 \%$ is omitted in the further discussion.

The age distribution of the participants was as follows: the mean age was 23.4 years, the minimum age was 17 years, the maximum age was 35 years, $1^{\text {st }}$ quantile was 22 years, the median was 24 years, and $3^{\text {rd }}$ quartile was 25 years. The distribution of different categories of exams is presented in Table 1. The data received is about 11 different exams from different domains. Analyzing inclusiveness of CBTs interfaces, followed by 
the effectiveness of various accommodations in CBTs and preferences of various accommodations among PVIs, is presented below.

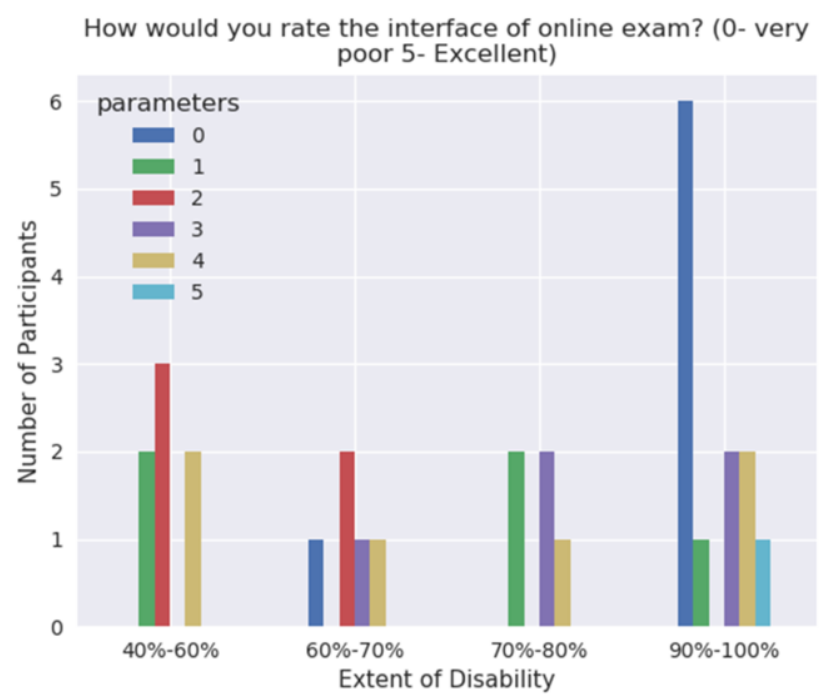

Figure 1. Exam Interface Rating

Table 1: Number of participants who appeared in CBTs

\begin{tabular}{clc}
\hline Category of CBT & Name of CBT & Number of Participants \\
\hline \multirow{3}{*}{ Post Graduate } & GATE & 7 \\
& JAM & 3 \\
& ICET & 1 \\
\hline \multirow{3}{*}{ Under Graduate } & JEE-Advanced & 3 \\
& BITSAT & 3 \\
& SAT & 1 \\
& KVPY & 1 \\
\hline \multirow{3}{*}{ Professional Recruitment } & Bank Exams & 5 \\
& Campus Placement Exams & 3 \\
& RRB & 1 \\
\hline & Govt. Recruitment Exams & $\mathbf{2 9}$ \\
\hline
\end{tabular}

\subsection{CBT Interfaces}

CBT interface is a combination of multiple components. The overall accessibility of a CBT interface is determined by the accessibility of individual components. To get a brief idea about the accessibility of a typical CBT interface, participants were asked to rate the CBT interface available during CBTs on a Likert scale. The responses can be seen in Figure 1. A total of 58.6\% PVIs rated exam interfaces two or below on a scale of $0-5$. In particular, $50 \%$ of the participants with $90 \%$ or more visual impairment gave 0 score to the CBTs interface. This shows a poor experience of PVIs with the CBT interfaces.

Figure 2 shows that more than $60 \%$ of participants faced significant difficulty (rating 3 or more on a scale of 1-5) in reading the upper-case words. This observation was consistent with the results in the research done by the cognitive scientists Arditi and Cho. [6] on the legibility of letter-case for PVIs and temporary able-bodied individuals. CBTs 


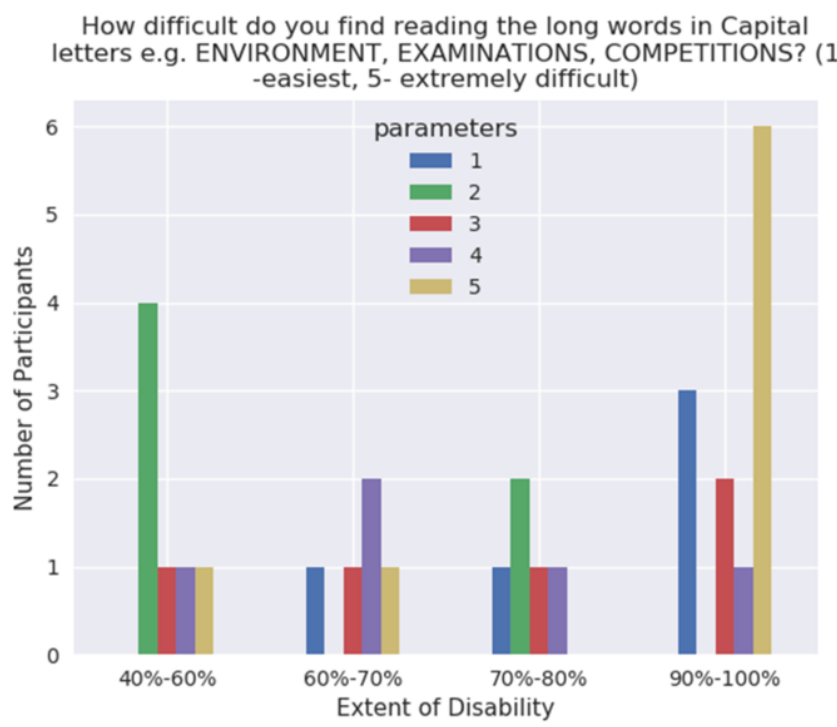

Figure 2. Reading the upper-case words

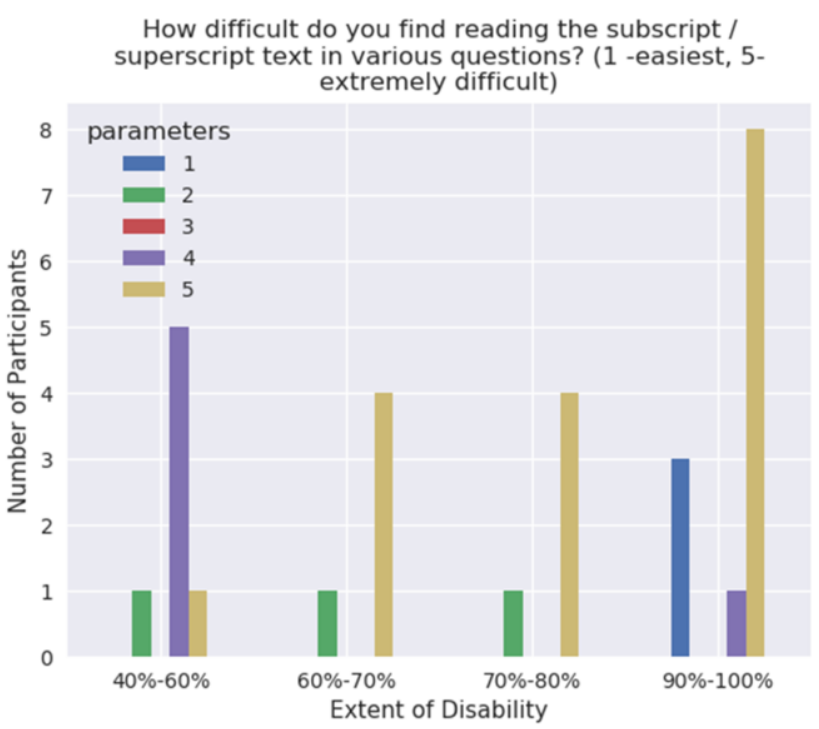

Figure 3. Reading the subscripts / superscripts

involving scientific formulae contain subscripts and superscripts. Figure 3 described the level of difficulty faced by PVIs in reading the subscripts and superscripts. It can be seen that a large majority of the participants reported 4 or 5 levels of difficulty, irrespective of their extent of disability.

CBT interfaces have other components apart from the questions to perform various operations such as selection of the questions, answering the questions, and operating the calculator. We asked the participants to highlight accessibility barriers of components in a typical CBT interface. Table 2 lists their responses. It can be seen that more than one- 
third of participants had some difficulty in accessing the calculator, images, and icons embedded in the CBT interface. Two participants reported barriers in accessing anything that requires movement of the mouse.

Table 2. Inaccessible components of exam interface

\begin{tabular}{lc}
\hline Inaccessible Component of CBT & Number of Participants \\
\hline Calculator & 12 \\
Images & 12 \\
Icons & 10 \\
Navigation using Mouse & 2 \\
\hline
\end{tabular}

Table 3.Common reasons for inaccessible interfaces

\begin{tabular}{lc}
\hline Reasons for inaccessibility & Number of Participants \\
\hline Inappropriate font size & 15 \\
Poor color contrast & 9 \\
Lack of screen reader support & 5 \\
Poor quality of images & 1 \\
Surrounding lighting conditions & 1 \\
\hline
\end{tabular}

To dig deeper into the reasons behind the inaccessibility of questions and other components of CBT interfaces, participants were asked to report common reasons for inaccessible platforms. Responses are shown in Table 3. We observed that inappropriate font-size used by the CBTs is the basic cause of inaccessibility for approximately $50 \%$ of PVIs. Since every PVI has their own font-size reading habits, providing options for changing the font-size of specific items could help eliminate one major cause of inaccessibility [6]. One participant reported a major issue that assigning a scribe for a CBT causes him/her to lose any advantage of having a computer-based exam. We quote:

"GATE 2018 did not have screen reader installed on their system. And they did not allow it to give it on my laptop. As a result, they provided me scribes. So it apparently makes no difference for me whether it was online or offline. Although the exam was computer based."

The participant raised an important question about the usability of a scribe in the CBT. Whether the exam is paper-based or computer-based, it does not make a difference to $\mathrm{him} / \mathrm{her}$ because, in either case, the scribe reads the content. This shows that the kinds of accommodations provided for paper-based exams are not sufficient for the CBTs.

To discuss the issues with accommodations further, we next analyze the most commonly available accommodations in the CBTs, which include magnification of text, scribe assistance, and compensatory time.

\subsection{Accommodation: Magnification}

To evaluate the effectiveness of magnification, participants were asked to rate the benefit of magnification on the Likert scale. The responses are shown in Figure 4. The effect of magnification depends on the extent of disability, as can be seen from the bar chart. Nine (09) out of twelve (12) people with 90 to 100 percent visual impairments reported that magnification of the text was barely useful because of less or no residual vision. On the other hand, 15 out of 17 people with up to $80 \%$ visual impairment reported reasonable improvements in reading speed due to magnification. 


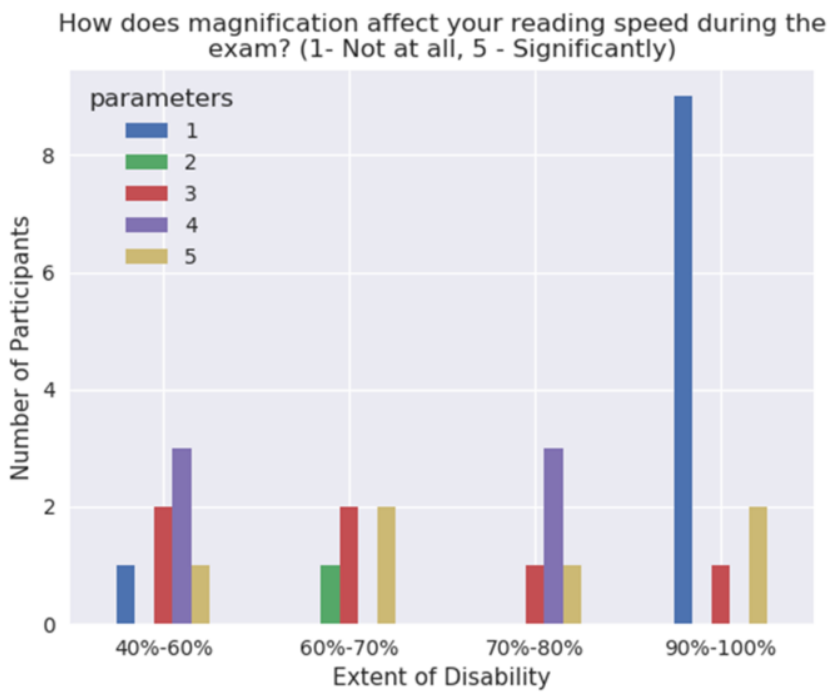

Figure 4. Increment in reading speed on magnification

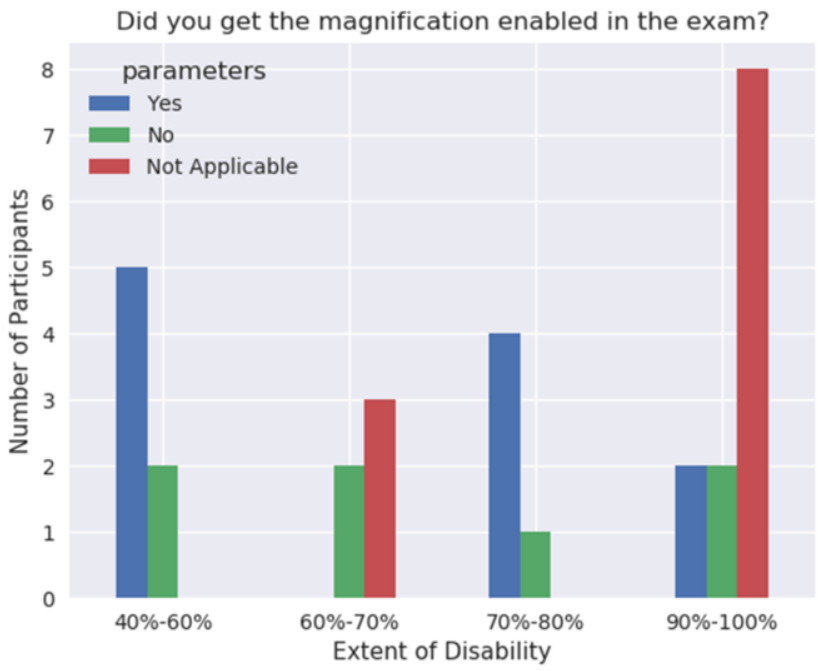

Figure 5 Availability of Magnification in CBTs

Our study contained querying about the availability of the magnification feature in the examination. As showed in Figure 5, only 37.9\% of the candidates reported that they got magnification enabled in the exam. $24 \%$ of the candidates did not get this accommodation during the exam, despite their need. Two major reasons behind this could be 1) CBT authorities did not manage to provide the magnification feature. 2) Participants had not asked for the same during the examination. It should also be noted that the candidates who did not ask for this accommodation were mostly in the category 


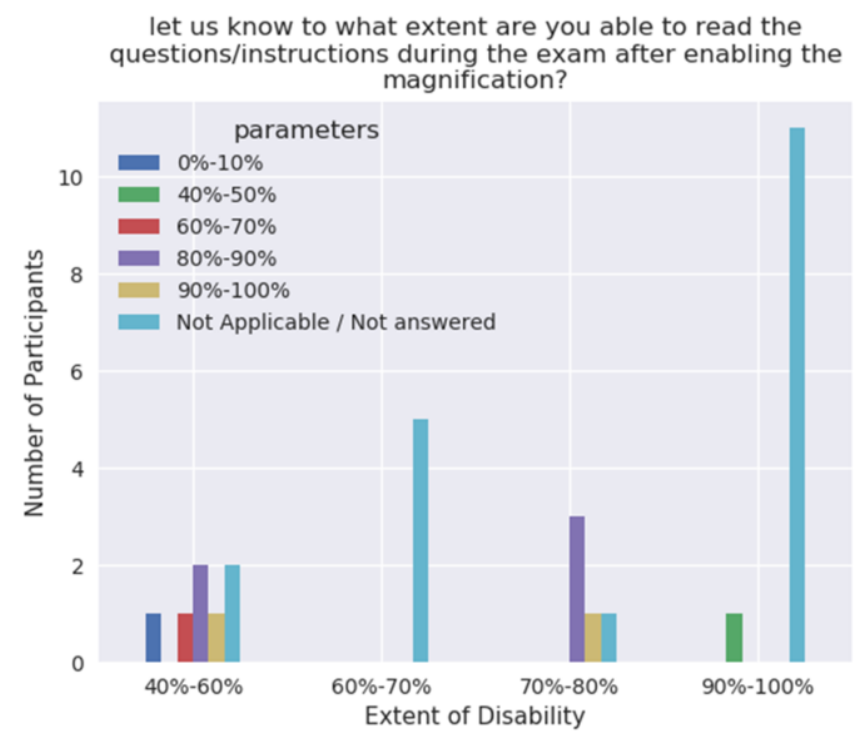

Figure 6 Extent of reading after enabling magnification

of $90-100$ percent visual impairment. As explained earlier, magnification as accommodation is of little or no use for them.

To evaluate the sufficiency of magnification, participants were asked about the extent of readability of questions after enabling the magnification. Their responses can be seen in Figure 6: $81 \%$ of the people who got the magnification enabled in the CBT were able to read up to $90 \%$ or less of the contents. This is not sufficient in today's competitive world, especially in an Indian context, where the drop of a single mark may lead to a low grade, rank in a course, or even disqualification from an entrance. Various reasons due to which participants are unable to read the complete contents, even after enabling the magnification, include insufficiency of the extent of magnification and heavy scrolling of text. The problem of heavy scrolling is identified by $90 \%$ of the PVIs, who got magnification enabled in CBT (see Figure 7).

To enhance the effectiveness of magnification for PVIs, a bigger monitor can be provided. This may reduce the need for the large extent of magnification and hence the problem of heavy-scrolling. This is confirmed by the respondents as given in Figure 8 . More than $40 \%$ PVIs opined that a bigger monitor setup could help them in easy reading of the text. On the other hand, $75 \%$ of PVIs with $90 \%-100 \%$ impairment reported that a bigger monitor setup is of no use for them as enabling magnification does not affect their reading performance.

We have seen that accommodation type 'magnification' was not useful for people with a severe (more than 90\%) visual limitation. Hence magnification is not a reasonable accommodation for such PVIs. Figure 5 to Figure 8 show that the majority of these candidates did not respond or opted for 'not applicable' to questions related to magnification. 


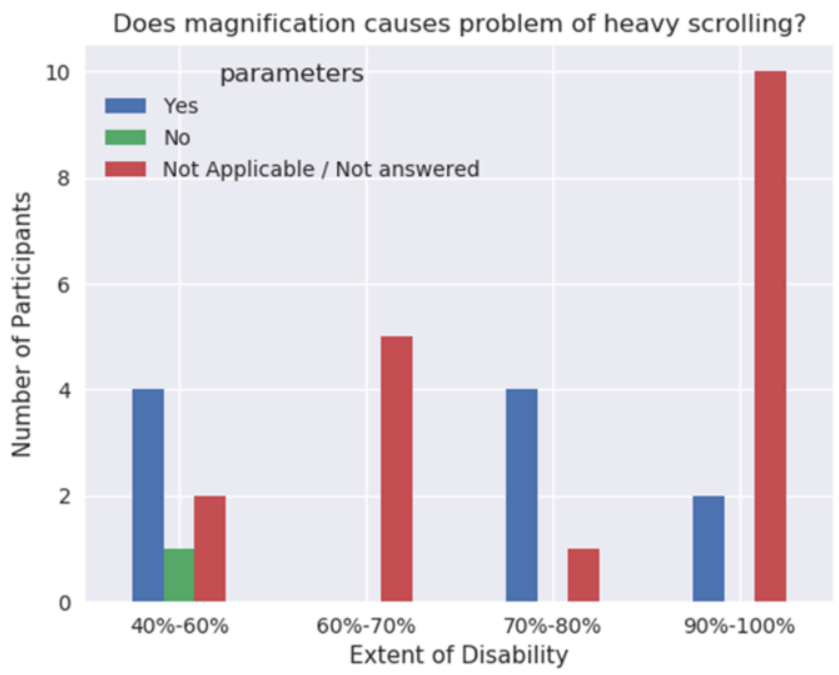

Figure 7. Heavy scrolling due to magnification

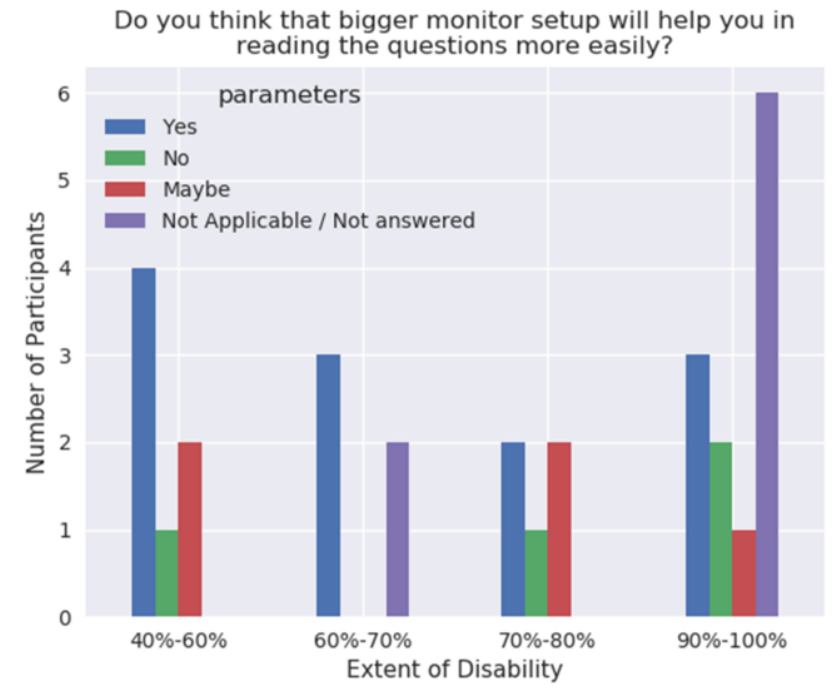

Figure 8. Possible effect of bigger monitor setup

\subsection{Accommodation: Scribe}

A scribe is another most commonly used accommodation in CBTs to provide equal opportunity to PVIs. Figure 9 displays the distribution of scribe accommodation used by PVIs. Out of the participants who have $90 \%$ or more disability out of these, $91.6 \%$ used a scribe in the CBTs. On the other hand, among the participants having $80 \%$ or less visual limitation, only $35.2 \%$ used a scribe in the CBTs. Participants with $90 \%$ or more 


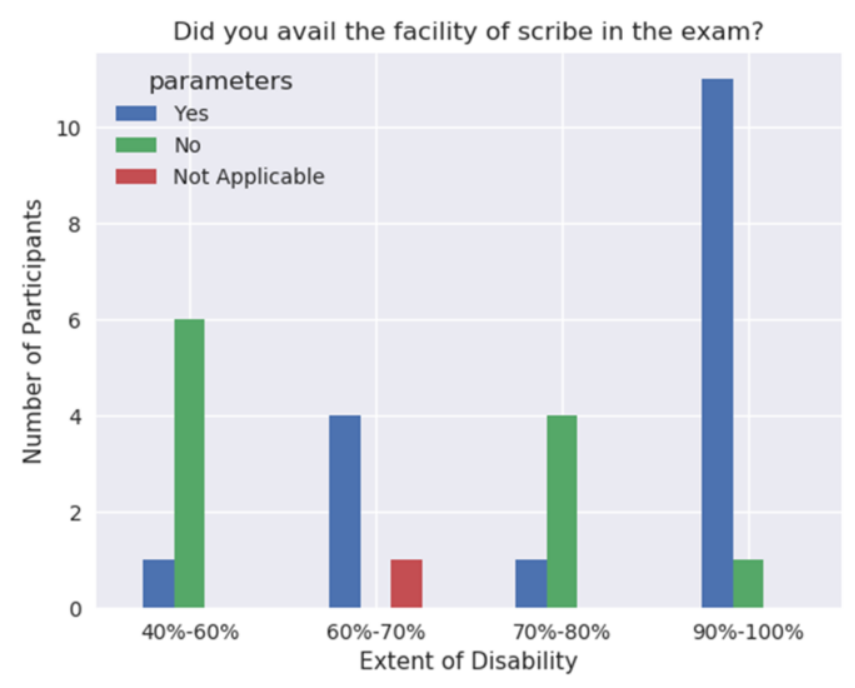

Figure 9. Availability of Scribe

disability preferred to use a scribe due to the lack of residual vision in their eyes. Hence they require assistance in reading questions/instructions during the CBTs. In contrast, participants with residual sensitivity in their eyes can read the information with enough magnification and/or appropriate color contrast.

In order to analyze the effectiveness of the scribe accommodation in CBTs, participants were asked to report the impact of scribe assistance on their performance in the CBT. It can be deduced from Figure 10 that only $27.3 \%$ of the participants, out of those who used a scribe, reported enhancement in their performance. The rest of the participants reported either drop or no impact on their performance in CBTs due to scribes. Considering the participants having $90 \%$ or more disability (Figure 10), it is easy to see that $41.6 \%$ of such participants who used a scribe reported enhancement in their performance, while $50 \%$ reported a drop or no impact on their performance. Of the participants having $80 \%$ or less disability, $58.8 \%$ reported a drop or no-impact in their performance, and only $5.8 \%$ reported enhancement in the performance. The above statistics about the effectiveness of a scribe in CBTs show that the scribe accommodation proves to be beneficial for a limited set of PVIs who have very less or no residual sensitivity in their eyes.

The discussion above brings us to a conclusion that PVIs who can read with magnification do not prefer to use a scribe for assistance in reading. To validate our reasoning, we asked the participants to provide feedback about their experiences with the scribe during CBT. The major concerns of the participants were as follows:

- The difference in the reading style of scribe versus the way participants expect to receive the information.

- The inadequate competence level of the scribe as per the examination requirement, to read the questions and notations with appropriate context.

- Language and accent barriers.

- Lack of practice with a scribe in the day to day life. 


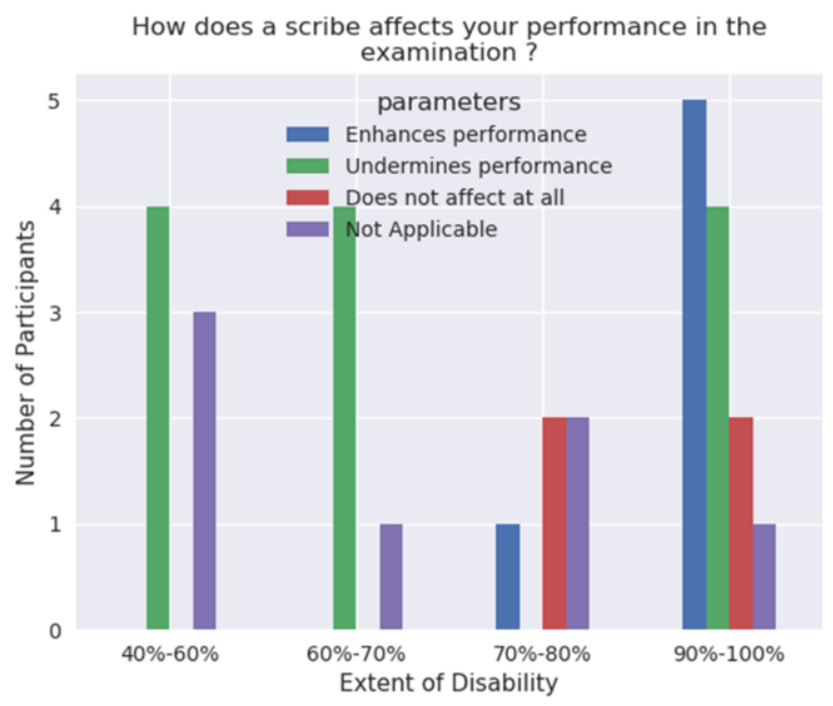

Figure 10. Impact on performance by utilizing scribes

- Lack of independence and control over the exam.

Qualitative responses from some of the participants who used a scribe during CBTs are stated below:

"Screen reader provides independence to re-read and navigate quickly as compare to scribe, especially for the long question, I am habitual of using screen reader as compare to scribe as I rarely get a chance to practice with a scribe."

"It's some time feel better to do to the accessibility of equations and other symbols, but assistive technology is better if there is good access."

"The scribe was less well versed with English and often did mistakes. He was also unaware of many maths symbols. Also as he was always looking into my paper I was losing confidence in myself."

"In normal day to day life, I am habituated to reading by myself rather than by a scribe. Hence it will have a bearing on the swiftness of understanding the question."

"Its hard to understand the code being read aloud to me."

\subsection{Accommodation: Compensatory time}

Compensatory time is an important accommodation that compensates for the time consumption in the CBTs due to various accessibility barriers by providing additional time to complete the exam. Intuitively, compensatory time would be a need of every PVI because of some difficulty in reading the question/instructions from the screen. We evaluated the sufficiency of standard time duration in the exam. As it can be seen in Figure 11, 86.2\% of the participants reported that the standard time duration was not enough for them to complete the exam. This evidence confirms our intuition about the 


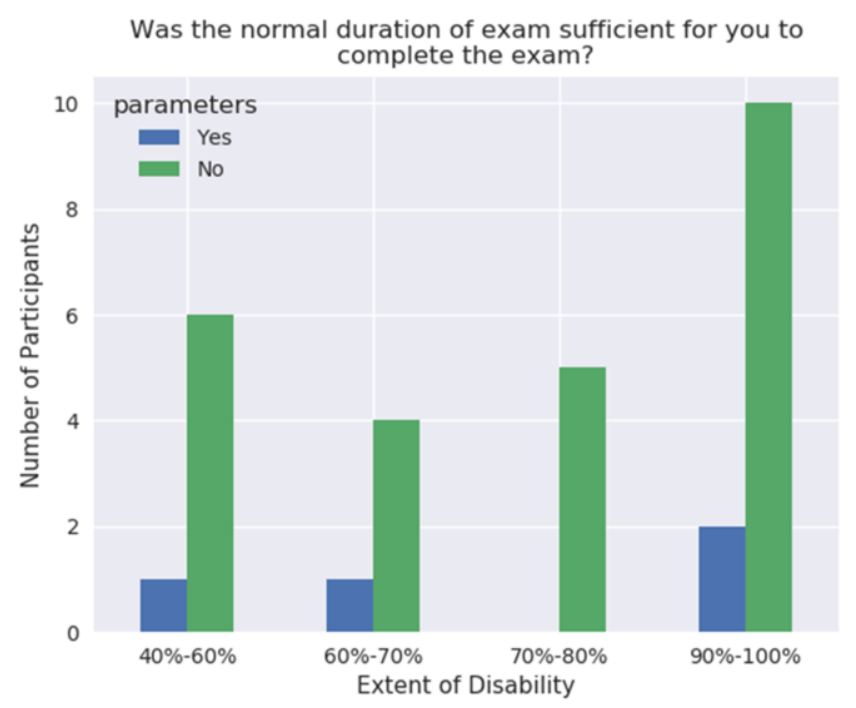

Figure 11. Sufficiency of standard time duration

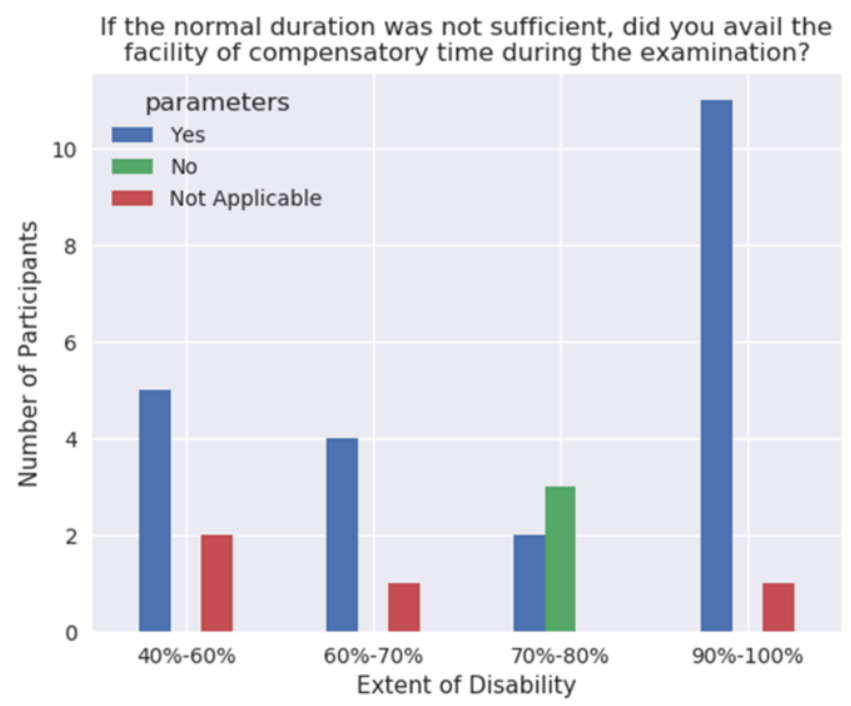

Figure 12. Availability of compensatory time

limitation of the magnification and scribe. In order to get more details, participants were asked if compensatory time was beneficial for them or not. From Figure 12, it can be observed that regardless of the extent of visual impairment, most participants used the compensatory time. A total of $75.8 \%$ of participants across the categories reported the same.

Figure 13 shows that $95.4 \%$ of those who availed the facility of compensatory time reported that it indeed helps in completing the CBT. 


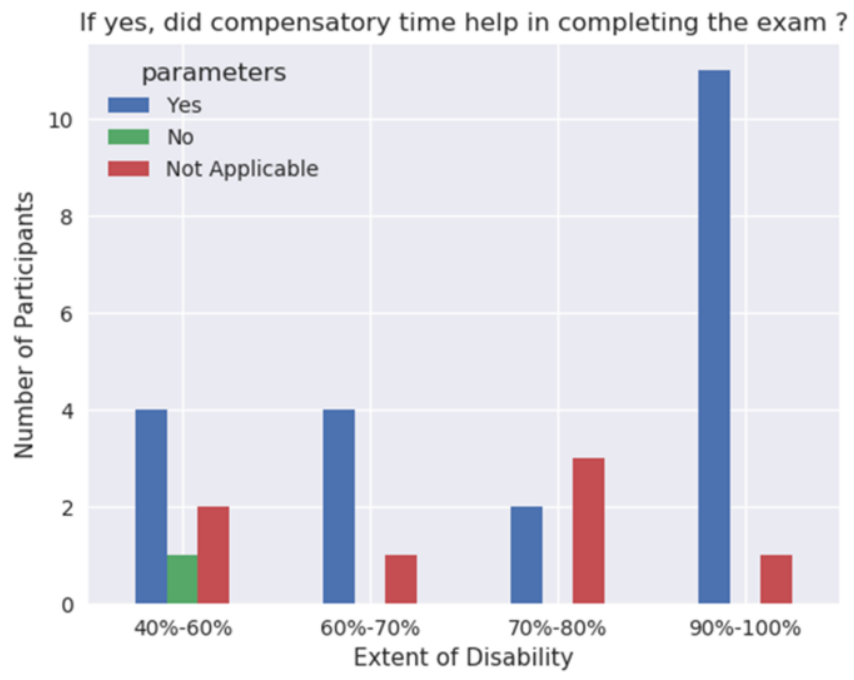

Figure 13. Efficiency of compensatory time

\subsection{Preferences among accommodations}

It was observed that different types of accommodations were suitable for different individuals depending on the extent of their disability. To further illustrate the fact, we asked participants about their preferred way of reading the questions. As it can be seen from Figure 14, 82.3\% PVIs having a visual disability of $80 \%$ or less prefer to read text with the help of magnification. Very few of them used a screen reader. On the other hand, $58.3 \%$ of PVIs having more than $90 \%$ visual impairment preferred to read with a screen reader. It is interesting to note that only $6.9 \%$ PVIs prefer to use scribe over other types of accommodations.

In reality, a single type of accommodation does not suffice to overcome the accessibility barriers in the CBTs. Hence, we made a comparison between the accommodations available, and accommodations expected by the participants. The responses were showed in Figure 15. Availability and expectation of scribe and compensatory time are remarkably similar, and these accommodations are used by $50 \%$ and $70 \%$ of the PVIs. On the other hand, accommodations such as magnification of text, screen reader, option for choosing color contrast, and bigger monitor setup were rarely available during CBTs; however, 30 to 50\% PVIs reported the requirement for these accommodations.

\section{Discussions and Recommendations}

One of the key goals of universal design is personalization. CBTs could consider specific and distinct accommodations as personalized adaptations or customizations for color blindness, complete blindness, and low vision. While talking technology through screen reading softwares are available, the issue of disturbance to other candidates 


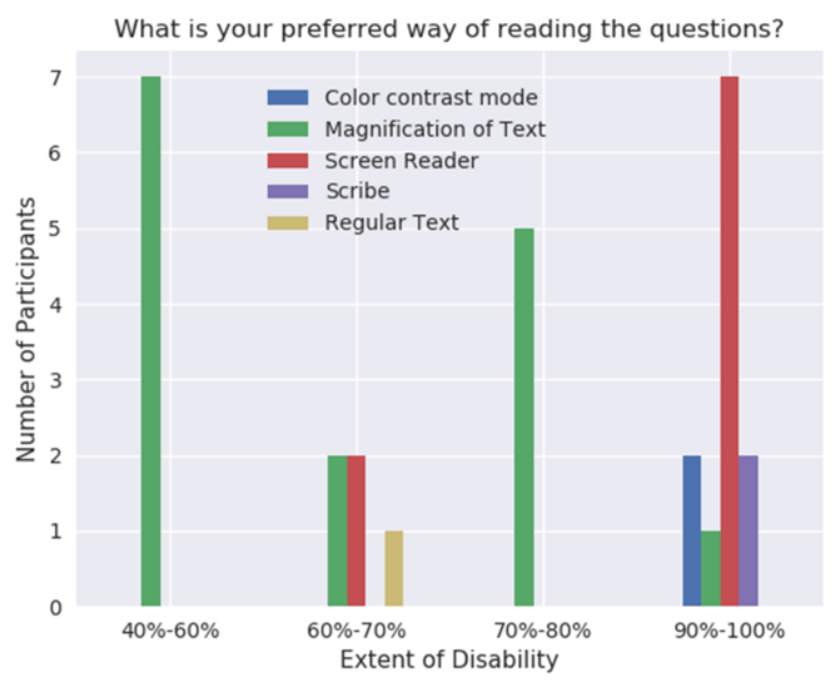

Figure 14. Reading preferences

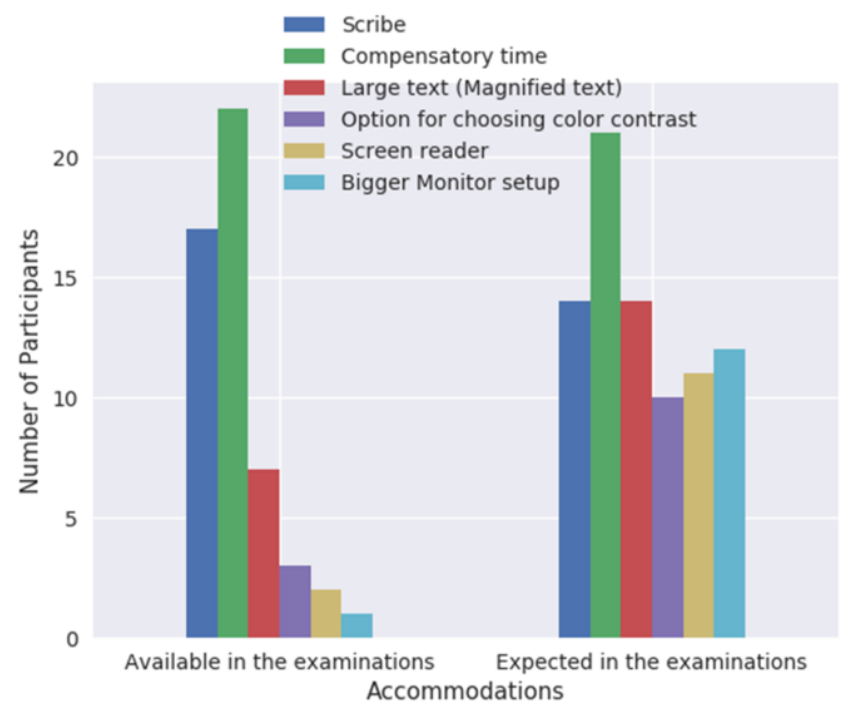

Figure 15 Available vs expected accommodations

remains a concern that surely can be resolved through mapping special need zones in the physical planning of CBTs. Alternately, the headphones could mitigate the same once vulnerabilities of technology are addressed, such as the threat of exam secrecy before and during the exam.

The color backgrounds and foregrounds, along with buttons in the objective type interface, need to be further studied for color blindness and visibility in different lighting conditions. Candidates with low vision or intellectual disabilities may also be impacted 
by the interface designs of different CBT platforms. The key principles of universal design as equitable use and tolerance for error need to be well integrated as guiding features of any test design. Considering an Indian scale of disability and complexity, CBTs need to be reviewed for diverse population groups for various exam types. It would be a dynamic exercise to evolve inclusive features in an incremental way in CBTs to make their reach wider and impact the idea of equal opportunity for all, including persons with disabilities.

Boundary push in CBTs could best resonate with the principles of Flexibility in use and enhanced Perceptibility of Information through added features for complete blindness, color blindness, and other disabilities. Recognizing the culturally and linguistically plural dimension of India, one could explore language translation feature as yet another attribute of a CBT reflecting the contextual layer of universal design and the goal of cultural appropriateness.

Below few recommendations are proposed to enhance the accessibility of CBTs and make them more inclusive. Technology-based assistance has a major role in the independent living [7] of the PVIs. It is preferred by PVIs because of global consistency in support and assurance of availability, as compared to human-based assistance (such as scribe) that lacks consistency (for example, having a different scribe for different exams). In recent years, with the advancement in assistive technology, PVIs are able to perform reading and writing tasks on their own, subject to the availability of the contents in an accessible format.

As shown in the research study by Web-Aim ${ }^{8}$, PVIs can experience different kinds of accessibility barriers due to variations in the causes of visual impairment. Hence there is a need for adaptive and inclusive CBT interface, which has the capability to personalize the CBTs as per one of universal design principle.

Since every PVI has a unique set of requirements for inclusive accommodations in the CBTs, so CBT conducting authorities should ask the PVI about their requirements for inclusive accommodations at the time of filling the application form. In order to achieve this, there is a need for the formation of an accessibility policy for CBTs, which entertains the accessibility and universal design of the CBT interface.

To improve the effectiveness of existing accommodations for PVI, below are the following accommodation-specific recommendations:

- To minimize the dependency on a scribe, the following technology-based solutions can be used:

o Use of screen readers such as JAWS ${ }^{9}$, NVDA $^{10}$.

o CBT questions in text-based DAISY ${ }^{11}$ format so that screen reader users can access the content.

o Providing CBTs in an alternate format such as computer voiced test similar to the one already available for GRE ${ }^{12}$.

- Highlighting: In our survey, 62\% PVIs reported average or more difficulty in reading the upper-case sentences and words. Upper-case words or sentences are used to highlight key attributes in the text. Here are some alternatives, can be used in the place of upper-case words:

\footnotetext{
${ }^{8} \mathrm{https}: / /$ webaim.org/articles/visual/

${ }^{9}$ Job Access with Speech - http://www.karishmaenterprises.com/JAWS.htm

${ }^{10}$ Non Visual Desktop Access - https://www.nvaccess.org/

${ }^{11} \mathrm{https} / / \mathrm{nfb}$.org/images/nfb/publications/bm/bm11/bm1102/bm110210.htm

$12 \mathrm{https} / / /$ www.ets.org/research/topics/assessing_people_with_disabilities
} 
o Increase the font size of the text

o Use of bold font-face

o Use of camel case words

o Highlighting of text background with different color

o Create a box around the text

o Underlining of the text

o Change of font-family can also be a good option for highlighting.

To achieve the objective of highlighting the text and low efforts in reading, a suitable combination of the above can be used. Since the combination of the above may vary from person to person, hence system should have the capability to set the appropriate combination prior to the examination.

- Magnification: Accessibility of various icons, subscripts, and superscripts in the text can be enhanced by providing two levels of magnification feature in the interface: one which zooms in the whole screen, and the other which zooms only a specific area on the screen to see very small fonts. Such magnifications are already available on popular operating systems like Microsoft Windows and Apple iOS.

- To avoid heavy scrolling due to magnification, a bigger monitor setup could be useful for low vision candidates. This could be of great help, as reported by $41.3 \%$ of the participants in this survey.

- The accessibility of digital calculators could be enhanced by re-designing them for screen reader compatibility or by providing a talking calculator [8].

\section{Conclusions and Future Work}

CBTs have paved a long way for inclusion and access to an Indian assessment system with its own set of challenges. In the growing expansion of the theory of universal design, these tests have already brought parity to include economic and cultural distinctions along with specific needs of persons with disabilities. The paper discusses the key barriers faced by students with visual impairments with the existing samples of CBTs. Interface design, font types, color distinction, scrolling, magnification, etc., need further investigations to find cross-cultural dimensions. Such studies need to be extended over a larger and more diverse sample group to further enhance its inclusive dimensions.

Advanced technology is being put to extensive use in Indian contexts for various CBTs. However, besides the specific challenges of the interface, the other broader challenges remain over the business models of infrastructure ownerships, ready state of physical infrastructure for conducting CBTs, and most importantly, the availability of accommodations to conduct tests in wide-spanning locations in India, instead of the current practice where the persons with disabilities are mapped to specific centers and locations.

It is important to highlight that Indian assessment systems for evaluations are leading towards a greater degree of contextual adaptations. The universal design approach surely holds the key for more holistic planning and enhancement of access in CBTs. This would include better integration of all disabilities, including individuals with complete blindness and other distinctions. Inclusive CBTs shall remain an important means to access in the times to come. With the help of assistive technologies, the CBT interface 
can be personalized as per the need for PVIs. The inclusive accommodations in CBTs should provide global availability, technological adaptability, and uniform consistency. These remain the critical dimensions and parameters to be researched to make accommodations in CBTs as inclusive as possible.

The study though an initial investigation is limited by a small number of participants. However, it paves a methodological structure to assess and evolve a direction to integrate a universal design approach in CBTs considering diverse disabilities and other cultural dimensions. To offset the small number of participants, the proposed recommendations are based not only on the feedback by the participants but also on the standard practices and manuals [7], [8]. Universal Design widens the horizon of inclusion and accommodation of other disabilities and diverse cultures towards a human-centric and adaptive CBT.

\section{Acknowledgments}

We thank all the participants of our survey for their voluntary participation and valuable time.

\section{References}

[1] H. Y. Abuaddous, M. Z. Jali and N. Basir, Web Accessibility Challenges, International Journal of Advanced Computer Science and Applications (IJACSA), p. 10, 2016.

[2] M. Hakkinen, Assistive Technologies for Computer-Based Assessments, R \& D Connections, Issue 24, June, 2015.

[3] W. Grussenmeyer, J. Garcia, E. Folmer and F. Jiang, Evaluating the Accessibility of the Job Search and Interview Process for People Who Are Blind and Visually Impaired, in W4A '17, Perth, Western Australia, Australia, 2017.

[4] T. Coughlan and K. Lister, The Accessibility of Administrative Processes: Assessing the Impacts on Students in Higher Education, in W4A '18, Lyon, France, 2018.

[5] G. Brajnik and S. Graca, On Accessibility Policies for Higher Education Institutions, in W4A '18, Lyon, France, 2018.

[6] A. Arditi and J. Cho, Letter case and text legibility in normal and low vision, Vision research, p. 19, 2007.

[7] L. B. Christensen, The Importance of Information Technology for Visually Impaired Children and Youngsters and the Expectations for Future Development, presented at ICEVI Krakov, 2000.

[8] XRCVC, I for Inclusion, The Xavier's Resource Centre for the Visually Challenged, 2014. 